Theoretical Physics, Astrophysics and Cosmology

Vol. 7, No 2, p. $21-37$, v1, 1 July 2012

Online: TPAC: 4200-026 v1, 18 September 2012
ISSN 1819-9135; ISSN 1991-3919 (e)

(C) 2012 CTPA. All rights reserved.

DOI: $10.9751 /$ TPAC.4200-026

\title{
Dialogues about a fate of stars: from black holes to frozars
}

\author{
Zahid Zakir ${ }^{1}$
}

\begin{abstract}
Sagredo and Salviati - two friends of Galilee - almost 400 years ago in Europe disputed about motion of planets, after which the heliocentric system have been understood and accepted almost by all. Their descendants, who were friends too, almost 40 years ago in America, disputed about a gravitational collapse of stars, after which the black holes have been understood and accepted, but not by all. Among not accepted were one of friends and as a result recently their dialogue has been continued in Asia, but already about a choice between black holes and frozars. After that frozars began to be understood and accepted almost by all who had read record of this dialogue and for this reason it is presenting for wider public also.
\end{abstract}

PACS: 04.20.Dg; 04.70.-s; 97.60.-s, 98.54.-h

relativistic stars, collapse, black holes, supernova, gamma burst, quasars

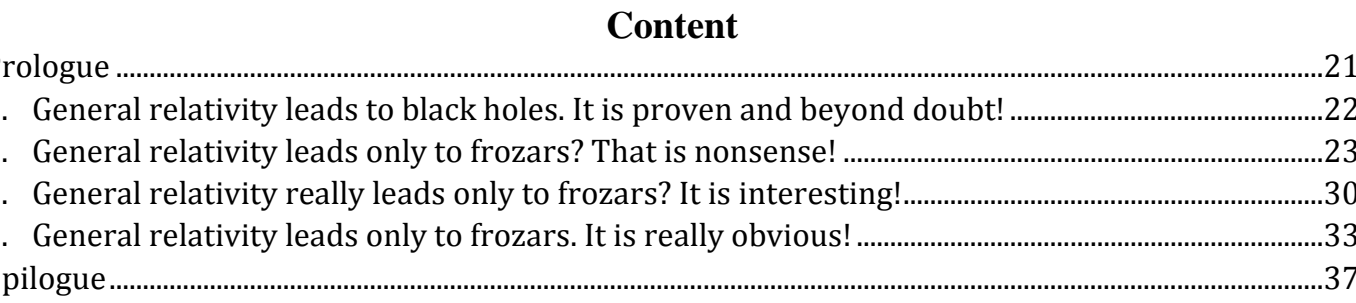

\section{Prologue}

Sagredo. If you remember, almost 40 years ago we had a dispute on a gravitational collapse when you have persuaded me that at collapse of an enough massive star a black hole should be formed. However, my doubts have not disappeared completely and during this time I have interested by this problem and have found new arguments. Are you ready to continue this dispute?

Salviati. Yes, I remember, but then I have proved for you that this is a strict consequence of the Einstein's general theory of relativity! Then many experts doubted also, but now this concept has gained a full and absolute victory - black holes are accepted by all scientific community and thousands of them are found out by astronomers. The term "black hole" became a part of global culture, it is applying in many spheres and known even children. So, what's a problem?

Sagredo. I mean't various possible holes where the blessings of civilization flow away. My objections connected only with the physical concept of black holes and its applications.

Salviati. So, I ready to return not to dispute, but once again to explain you this outstanding

${ }^{1}$ Centre for Theoretical Physics and Astrophyics, Tashkent, Uzbekistan; zahidzakir@theor-phys.org 
theory about the most amazing objects of the Universe and to tell about new successes in this most exciting area of cosmic sciences.

Sagredo. You have not paid attention that I have told - I know everything that you will tell about them, because your favorite concept have not changed since and then I also read almost all that, as you. Now dialogue will be not an expert with a beginner, but now the present dispute will be on equal conditions.

\section{General relativity leads to black holes. It is proven and beyond doubt!}

Salviati. I did not expect from you such, honest, I thought better about you. Though, why not to dispute, especially, when a result is in advance obvious to me. But the conditions there just unequal - on my side all scientific community and ten thousands publications, hundreds conferences and, eventually, destiny of thousands people which almost all life developed different aspects of this outstanding achievement of the twentieth century science. Huge literature for the reader of any level, set of films, thousands TV programs reported about black holes. In the Internet you can find millions references to this term, hundreds discussions on tens languages. So, it could be better, if you could study something from this even more carefully before to start serious dispute. Otherwise we waste our time and about you there will be a bad reputation of a person, contrary to all trying to reject reliably confirmed scientific theory entered into the gold fund of mankind and obvious even to any student.

Sagredo. As I have already told, I have satisfied in advance your conditions and consequently already not you for me, but I can bring you over regardless of a temporary victory of your favorite hypothesis and its worldwide promotion in unprecedented scales. At that time I did not understood much in refinements of GR, while you were worked with it closely and then I have believed to you. Since then I have studied all the best literature on this problem, besides it is not so much textbooks, where proofs contain "white spots" and are often similar on swinging by hands in front of trustful students, but studied works of founders which have not been so categorical and confident, as their followers, watched later scientific literature also. I have found out with surprise, and you also well know, how even founders of GR, who hesitated in a choice of variants, ambiguously applied and quite often preferred just on formally correct, but physically mistaken versions. Thus, my doubts in a consistency of the black hole hypothesis have proved to be true and now I precisely know that this hypothesis does not follow from GR, the interpretations of observations are unjustified and for them give out the observation of completely different nature objects.

Salviati. Oh, that is the question! If I did not know you personally would not to continue this talk. When I obtain messages with statements that the black holes do not exist, I do not read them further and the authors I carry to "persona non grata" for my mailbox, on their doubtful articles with elementary errors, submitted to journals, I give a negative response, and at conferences and seminars I do not recommend to put their reports in the program not to waste listeners' time.

Sagredo. My qualification not so differ from yours, thus if knowing it you will ignore my opinion too - you can't do the impossible for you - this means that you are engaged in everything, only not in a science.

Salviati. But you well know that objections against the black holes were hundreds, thousands high quality professionals tried to build alternative models more than eighty years. Even Einstein has tried, but cannot bring clear arguments in favor of the factors keeping the fated star from the collapse in the gravitational radius. Many have tried to overcome this hard nut - 
after all the author of the term "black hole" named the gravitational collapse almost greatest crisis of the twentieth century physics. But it has been then proved that all troubles are reliably covered under horizon in a black hole and the crisis has been overcome. So, for me this question is closed once and forever, as I advise for you too. I do not wish to continue this pointless dispute which is equivalent to attempt to deny GR, all predictions of which brilliantly proven during almost the century, and cast doubt on the astrophysical data about thousands discovered black holes.

Sagredo. I suggest to you to continue that scientific dispute where you apparently have convinced me because of my inexperience and naivety, and you say that it already selfevident truth. If you are so confident to it, what prevent you to deny my new arguments too? Usually erroneous hypotheses, which have begun to be standard paradigm, keep basically on trust to authorities and that many have not got used to exploit own head and according to its intended purpose.

Salviati. Well, I am ready listening your arguments, but only to desire to help you open your eyes. So, what is a reason to your mistrust to this theory? You have started to accuse it even in quasi-scientific character! Why?

Sagredo. Even if while we have not concerned it, but as you praise it to the skies and fence off from criticism. First, at beginning you do a doubtful statement that black holes are a unique and strict consequence of GR, and then give up all arguments even in frameworks of GR against this idea, being covered with authority of this theory and a demagogical phrase that it is attempt to deny GR. Secondly, observation of the objects, nature of which while is unknown and there is no any evidence of their internal structure, you loudly and confidently attribute to discovering desired black holes alleging that there cannot be anything else. Thirdly, you without knowing and without trying to understand, not to discuss, my arguments against them, confidently are doing conclusions to own advantage. That is a scientific approach?

Salviati. I do not wish to start this dispute to keep our friendly terms only. You like to analyze and very critically think, but for all there is a limit.

\section{General relativity leads only to frozars? That is nonsense!}

Sagredo. Oh yes, years have changed you and, it seems, I turn to the wrong door. It is a pity that dispute will not take place. But before we will leave everyone at its opinion, I would like to clear up impressing statistics presented by you. First, if from several hundred alternative approaches to the collapse to leave only those which do not fall outside the standard GR, then there remain about few tens approaches. Secondly, if from these remained to exclude also those one in which were postulated unknown forces, particles or mysterious forms of matter or energy, all set of realistic alternatives is narrowed to three-four ones. Thirdly, these few reliable alternatives investigated not too many researchers and they, unfortunately, having started to move in a correct direction, made hidden or visible errors, by leading itself at a loss and, that is worse, compromising in the opinion of others these almost right ways.

Salviati. It not seems to you that for a correct theory these three-four equivalent alternatives are too much. Unlike this small and confused scientific opposition, the theory of black holes is unambiguous, beautiful, mathematically rigorously proved and widely confirmed by observations. Thus, let we will leave on that point - I really hurry up on a large international conference on the internal structure of black holes in one of the best touristic centers of the world and after arrival I with pleasure will share with you about new facts about really existing black holes. Though, if you take into your head this crazy idea that black holes do not exist, the observations perhaps cannot bring you over and for this reason I much regret that 
you begin to lose the sense of a reality.

Sagredo. I did not say to you that all these few alternatives are equivalent. All of them had contained one a common true element, after which then there began variations and conjectures. These ones were doubtful only. But, I was convinced that recently there remained only one a consecutive treatment of the relativistic collapse which is clear and strictly follows from GR already without any hypotheses. It is the theory of frozars - relativistic stars with frozen matter and internal structure. Are you known about it?

Salviati. No, and I do not want to know, since the treatment of the frozen stars is not new and it is an overcome stage already for a long time. It is doubtful that you had studied the literature.

Sagredo. Well, about it we will talk later, and do not forget that all new is a well forgotten old, and such new appears as a well-grounded if we come back to it after long time trials and mistakes. In regard to your trip I doubt that you there find out something new, except touristic delights because the structure of your black holes is simple, for a long time is described in any textbook, and observations, according this hypothesis, are absolutely useless in the question on their internal structure. External effects of compact stars, which have passed at collapse into the frozar state, in simple cases almost the same, as your black holes. Since only these outer effects only are accessible to the observations, everything that to you will tell about thousands already found black holes, in fact I now assign to thousands already found frozars and I advise to you to treat in that sense. I wish to help you begin to look to existing hypotheses more critically and not to be too trustful - we have a common purpose irrespective of personal predilections and both we are interested in how these compact stars are actually composed, are you agree?

Salviati. So-so, but, honestly, we have a strange dialogue - everyone said own and we did not listen each other. Meanwhile we have agreed only in mutual recriminations in quasi-scientific character. And here, allow me to remind to you, that you accuse of it almost all scientific community whereas your point of view, which you strive to impose me, adheres, except its author, still only one and a half of its followers - whether it seems to you it is doubtful and even a little pretentious?

Sagredo. About a numerical superiority at present you are, of course, right, but how many followers there are at appearance of any correct idea incompatible with standard one? Remember history of any important consequence of GR before its confirmation, for example, Universe's expansion. Then even Einstein has strongly rejected this idea and only after some time had agreed with it, admitting that make a mistake, having trusted too to intuition, instead of the equations of own theory. In a case frozars the situation has appeared the same - they follow from the equations of GR, while the black holes are dictated by intuition hidden following on the Newtonian theory. For the science in such situation it is important and decisive not a number of supporters, which trust those who is engaged in it, but something another - scientific scrupulousness of these people. I well know both versions - and with black holes, and with frozars, - while you know only one version and do not want know about the second which in essence has not been properly developed when authors, to whom you trust, wrote their well-known articles and books. Thus, don't count your chickens before they are hatched. For me, the unpretentiousness in science means to be adequate to the reality, so it is really pretentious to attribute just to the huge real world and to impose to all mankind that seems too much true and about what have simply agreed, but that is realized never. Always it is easier to evade from criticism, than to answer it in essence.

Salviati. I do not evade on your criticism or your some author. Simply it is expensive pleasure to waste time and forces on those who doubts on the worldwide recognized theory. You simply do not represent and do not understand in what scales it is recognized - the black hole theory is toughing in more than ten thousand universities of the world by ten thousands 
professors to millions students, it is stated in hundreds millions copies of books and journals and is showed by TV and films to billions viewers! Many billions dollars are already spent for check of its consequences and all it simply mistake?

Sagredo. The worldwide propaganda of black holes has given much for the development of astrophysics and it is the fact. Many results concerning the phenomena outside the gravitational radius keep the force - and it is almost eighty percent of all scientific publications and demonstrations for public - and it is the fact too. But everything concerning fantasies about unobservable internal structure of black holes is a grandiose misunderstanding and one of curious legacies of such changeable and ideologized century as the twentieth and it is necessary to avoid this legacy quicker. You know that mass acceptance of a hypothesis yet does not mean its validity - mass propaganda often does not based on a scientific argument remember, how many there is fascinating feature tale films about a time machine moving you in the past, allowing you to change something there. All these jumps on millions light years through wormholes faster light velocity, are propaganding by prominent scientists fully seriously, are not better them.

Salviati. Sorry, if you accuse in ideologization, it becomes already a little awkward to me where you see all it? I do not see. The concept is simply so paradoxical and its consequences so grasp imagination that millions people with pleasure are interested in these objects only. While I agree that it's not bad idea to listen your arguments even if they are denied before thousand times and it is better than falling out. I see at you indications of beginning megalomania and you should be cured of it - after all you declare that you know unique rights answer and that all scientific community and this huge world audience are mistaken. Well, please, I am ready to discuss these yours frozars or as you still them there name. Please, only not to take offence, if I utterly demolish them.

Sagredo. I am glad for you and I congratulate - you have just passed exactly half of way on finish of which becomes a supporter of the frozars theory. After all you have broken the main barrier on this way - psychological, and get ready, though with a prejudice, to look into the theory rejecting your black holes. So, you will see, what of treatments will be defeated utterly.

Salviati. Well-well, I begin to envy your enthusiasm, though already it inspires fear that you have simply allured something and, probably, time would make you able to think clearly, but I will make it faster. For now, please, let's begin with in what this treatment coincides with the former concept of black holes and then already tell, in what is distinction and why and what follows from it. Are you agreeing?

Sagredo. Quite. In the black hole theory the collapse process passes two stages - before and after (including a moment) crossing by a surface of an object its gravitational radius where the falling speeds reaches the light velocity. And so, the frozars theory consists of standard applications of GR to the first stage of the collapse in terms of world time (time of distant observers) plus the statement that the second stage of the collapse, predicted by the Newtonian theory, in GR is absent. As you can see, at the first stage of collapse two treatments are in essence identical, since the same equations GR with the same standard (direct or numerical) solutions, only the accent becomes on other aspects. The most of predictions of the former concept of black holes about the phenomena outside the star's gravitational radius remain the same with small specifications. That's all.

Salviati. Not so much, though now I have already a little settles down. As I understand, in this treatment all observable consequences of the former concept almost remain and the dispute will go basically about different treatment of unobservable internal structure of the collapsed objects.

Sagredo. In general, yes. The internal structure of frozars is essentially different than black holes and it is in principle observable, though is much more difficult and rarely, than outer effects. 
Salviati. Then discussion passes to a pure theoretical area and here you are trapping. After all there are mathematically strictly proved and became famous theorems about inevitability of singularity in GR, when all matter of a star very quickly (on proper time) falls to the centre and becomes contracted into a point with infinite density.

Sagredo. The matter is not about mathematical severity of these theorems, but that they have been proved at a condition that "if object's surface crosses its gravitational radius, then ..." and so on. Thus, this "if" is natural in the Newtonian physics, but as it is shown in the frozars theory, has place neither in GR, nor in the real world. The surface of objects never crosses their gravitational radius not only in world time, but in proper time too.

Salviati. That is an error. In GR the surface does not pass gravitational radius only in world time, but crosses it without problems in proper time and this fact lies on the basis of the black hole theory at all.

Sagredo. For this reason world time has been treated as a "bad" time coordinate and proper time as a "good" one, so?

Salviati. Of course. A true time expressing a natural course of physical processes in each point is proper time, intervals of which in GR are invariant. Therefore, world lines of particles on the surface easily proceed in the gravitational radius in terms of proper time. World time does not express of it, so it is really unsuccessful choice of coordinates.

Sagredo. Here now we are also returning to distinctions of two treatments - in the frozars theory it is proved opposite that proper time is a bad choice for time coordinate since only world time expresses a true course of simultaneous coevolution of all parts of a star in coordination at global scales with temporary evolution of the real world.

Salviati. Thus, in this new treatment the situation with times is opposite - proper time is bad and world time is good time coordinate, is it right?

Sagredo. Yes so. There are strong reasons for this in frameworks of GR.

Salviati. Well, this point of view, at least basically, has a right to existence, although I do not understand, what then you will do with the second stage of collapse? You will simply deny it only because it is absent at your special choice of coordinates? I doubted something that. Usually many opponents of black holes reduced their proof to any private choice of coordinates and then inflated value of this arbitrariness. In the black hole theory the choice is made in favor of physically dedicated and invariant coordinate - proper time - where arbitrariness is excluded by definition.

Sagredo. Yes, you are right under the relation to many former alternative treatments, but not to the frozars theory. In GR both time coordinates express two physically equally essential sides of temporal evolution and consequently both are physically dedicated. Thus, to overcome worries in a trivial arbitrariness, let's specify the definitions.

Salviati. Really, give exact definitions; otherwise we can wander to anywhere.

Sagredo. An essential point is that time has two basic physical properties - a rate of its course at any point and a simultaneity of events at different points. Both these properties of time are important and fundamental. In the Newtonian physics there is no need to distinguish them, while in GR it is not so. In GR an event along a world line of particles on surface and interior of a star at the first stage of collapse is parameterized by two times - proper time and world time. The first defines rate of course of the local processes, the second - simultaneity of these events at different parts of the star and with distant events. In this sense the parallel description in world time gives a physical meaning to the moments of proper time, by adhering them to a certain epoch of the big real world into which the star as a whole is embedded. Thus at the first stage of collapse what of these two kinds of time you use, formally is a question of convenience and statement of your problems - both of them are unequivocally connected with each other for any event. Are you agreeing? 
Salviati. Yes-yes, all those are known things.

Sagredo. Advantages of proper time, as you have told, that its intervals invariant and have the direct physical meaning. But proper time - a bad time coordinate since its rate, which is slowed down by gravitation and the speed of falling, different for different layers of the star and at contraction sufficiently varies even for the same layer. As a result, for the given layer its intervals are not equidistant neither with respect to world time, nor to proper times of other layers, even for the same layer at different moments. Main disadvantage of description of evolution on proper time - on its moments in different layers it is impossible to define a star as a whole extended object the parts of which coexist simultaneously.

Salviati. I agree that at using proper time one must be very carefully.

Sagredo. The physical simultaneity of events in the star, by definition, is given by the moments of world time. Only by collecting the events with the same value of world time we can attribute them to separate parts of the star coexisting simultaneously and, consequently, forming at this world time moment the such extended object as a star.

Salviati. While you are right only in that in the black hole theory the simultaneity of events does not taken into account and there do not given such great significance to this aspect of evolution of a star, as you try to inspire it me.

Sagredo. The real world, as it is known, is a set of simultaneously coexisting objects. In GR there are many complications with a simultaneity of distant events, but in any case when it is possibly to define a global homogeneity of events, as in the case of spherical stars, you must to define evolution of extended objects as a whole as their "photo-pictures" at certain moments of world time. Any other picture does not concern to the real world and is only a mathematical fiction only. Are you agreeing?

Salviati. All that is true in special relativity, but in a static field of a spherical star, it seems the same situation. Do not forget also that in moving frames the picture changes.

Sagredo. Not it seems, but exactly the same, though with some precautions at synchronization of clocks due to slowering of light speed in strong fields. A motion of observer's frame does not change a physical picture of star's evolution, so it is enough to describe this evolution in the rest frame of star's centre where no artificial kinematical complications. Therefore, in the black hole theory only the first stage of collapse is described truly, though very quickly and not enough clearly. And here the second stage of collapse, when proper times have been supposed as unrelated with world time and are forced to proceed independently, does not concern to the real world where all objects coexist at a definite moment of world time. Here in fact a bad and distorting a true course of collapse temporary coordinate is proper time. World time both was and remains as a good temporary coordinate for all time of existing of a star in the Universe.

Salviati. So, good clock is not which counts my heart's beats, but which shows world time. I have no objection.

Sagredo. When you will froze with fright or, sorry, die, your heart clock will be slowing down or stopped, while your world clock continues to go. The star's collapse in fact is its death, so the black hole theory is based on the description of processes in a rate of heart beats of died, which is absurd.

Salviati. As soon as you begin to give detailed definitions, distinctions became clearer and terrifically obvious. Thus, while we have not got confused in details, give all begin one after another. On what this new theory of collapse is based and to what it leads?

Sagredo. Yes, further it is better to define more exactly main statements of the theory of frozars. First, we work in the frameworks of the standard GR without hypothetical additions and, consequently, we take standard solutions of the Einstein equations around a star and material solutions in its internal region with standard model simplifications. 
Salviati. It is very well that you are adding nothing to GR. It will be easier to deny you. Usually most of oppositionists are protect their hypotheses from a direct logical refutation by cleverly adding some details which cannot be checked up in further millions years.

Sagredo. Be quieted, this approach is simpler and clearer than all former ones and is not based on any artful "inventions". All secret just consists in cleaning of the theory from the erroneous complications of former attempts to describe collapse and nothing any.

Salviati. I doubt that. Most likely erroneous is your statement that conventional trues are erroneous. So, what further?

Sagredo. Secondly, we consider collapse of an ordinary star only with ordinary matter and known forces and thus initially its surface is placed outside the gravitational radius.

Salviati. Here again add nothing? And in vain, here you could allow yourselves some undetectable forced, darker matter or energy which can be found out never, and an alternative to black holes could be ready! Mainly, it could not be deny in any way!

Sagredo. I interested, as I hope you too, by not fiction worlds which are uncountable, but by the unique real world, for description of which in the case of collapse there it is enough GR in its simplest form, so I continue. Thirdly, we will suppose that at the first stage of collapse at any spatial point the time of events is marked on by two types of clocks - proper time by the standard clocks and world time by the coordinate clocks. Besides the rate of proper time is slowed down at different places differently and as more than gravitation is stronger. There the coordinate clocks are fastened with respect to the standard ones so that everywhere to be synchronous with distant observer's clocks. Are you agreeing that all that follow from GR?

Salviati. Yes, of course, while you state standard things. I do not see anything new. Only you still inflate the first stage of collapse though in GR all it is elementary and clear.

Sagredo. Excellent, we though in something have started to reach the consent. Key for the frozars theory, I have specially specified in detail the statements necessary for further, that you should not think that something new and doubtful is entered. Moreover, I am glad that all it is elementary and obvious for you - it will be easier to you to apprehend why from GR follow frozars, instead of black holes. Further, it is clear that the same general conditions in both treatments in some cases lead and to the same consequences.

Salviati. Therefore, if in consequences also there is no difference, you straightforwardly should come to black holes! It turns out, much ado about nothing?

Sagredo. Do not hurry up and do not generalize - I say about the same consequences not in all cases, but in some cases.

Salviati. Well, then specify these conditions and I will show, where a mistake in your reasoning, because if you have done all correctly, you should be come to black holes and nothing else. So was with all who seriously investigated this problem, so it could be with you too, if you have not blend something.

Sagredo. At all your self-confident irony, I catch you at expression "if you have done all correctly". Why you so confident that in the black hole theory all have been "done correctly"? Let us I specify what I mean under this expression to overcome usual misunderstandings about it. You are doing all correctly if, by starting on the defined initial conditions and no adding anything contradicting them, work in our real Universe with its averagely regularly going world time, at any instant of which all physical objects simultaneously coexist. For our collapsing star at each moment, both on world time and correspond to it proper time, we proceed length of perimeter of star's surface and internal layers. Have you objections to that?

Salviati. You, as before, continue to inflate significance of the simultaneity and world time describing it. In the black hole theory, as well as in GR as a whole, a true time in which any object evolves, is its proper time. Any student knows that in terms of proper time collapse 
occurs exactly as in the Newtonian theory, the surface passes gravitational radius without problems and all matter very quickly falls to the centre by forming a singularity, an infinitely dense state, though for a distant observer on its world time object's surface seems frozen forever outside the gravitational radius. You at first wish to replace proper time by arbitrarily entered world time and then declare this arbitrariness as a unique correct description? I tell at once that if it is your entire secret, this trick will not take place, since that is not a case of GR which allows entering times of different observers, all of which are relative and only proper time of the object is invariant.

Sagredo. But you have agreed that before the surface could cross the gravitational radius, any proper time moment on the surface is uniquely connected with a certain moment of world time and the descriptions of surface's motion in terms of both times are equivalent? You, certainly, also well know, that in GR at any world time moment corresponding moments of proper time of spherical star's surface also are uniquely related with the proper time moments of internal layers up to the centre?

Salviati. At moments before crossing by surface the gravitational radius, of course, I agree. Though both times for the description of trajectories basically are absolutely equivalent, but in proper time a picture is sufficiently simpler. But, as surface crosses the gravitational radius, you must, want or not, to enter the more reasonable coordinates.

Sagredo. So, you perfectly know that outside the star and on its surface the moments of world time have direct physical meaning too and mark events simultaneous with the corresponding moments of proper time of distant observers - after all they are synchronous with clocks of the last ones. Simultaneity of distant events - here is in what world time is physically singled out and it is also important and objective as invariance of proper times. If remember, special relativity has begun with Einstein's definition that "all our judgments in which time plays a part are always judgments of simultaneous events". Notice, you agreed that in the rest frame of a spherical star the simultaneity of distant events is defined as in special relativity.

Salviati. Well, of course, but only until the proper time moment when surface crosses the gravitational radius. After that you cannot say about any simultaneity - the star falls into the events horizon, whence already any signal does not leave. You in vain do such importance on simultaneity - in GR in each point generally it is possible to set any coordinates and simultaneity of distant events there is not a rule, but an exception and a big rarity.

Sagredo. But you perfectly know that exactly this rare occurrence and takes place around and on the surface of a spherical star and that here the global simultaneity of events takes place!

Salviati. How many times I have told that yes, and let's close this theme, if not my patience already bursts! It is simply impossible, why you're wasting your and my breath and time for these elementary matters which one set to students as easy problems?

Sagredo. When Jupiter became angry, all knew that he is not right. But it was for a long time. Are not heated, we are already close to finish and almost everything that is necessary, have already specified.

Salviati. Yes? I have not seen that you frozars appear somewhere - where they are? Perhaps, as in a focus, now you will take out them from a box with a secret?

Sagredo. For any civilized man his box with a secret is his computer and from it he can to take out and show on screens everything. In any good detective it seems that in vain deal with boring details and terrible garbage - with some things, pigments and so on. Archeologists at archaeological site too long dig out something unclear pieces and then start to build chains of reasoning, restoring a full picture on these slices of a mosaic.

Salviati. Well, when you will begin this "chain" as you say, "reasoning". While, as I see, dig is not deep and results, perhaps, will be the same.

Sagredo. Before to pass to this logical chain, let's specify once again with what from the 
details necessary for this purpose you have already agreed. In standard GR we are studying a standard (spherical and non-rotating) star, fuel of which has ended and which begins to contract gravitationally and the surface already close to the gravitational radius.

Salviati. If you take all absolutely standard, give for simplicity a thin dust shell, where no interaction of particles and there was only gravitation, so the problem is exactly solved. And then pass to a dust ball which is more difficult, but there are well known exact solutions too. On these simple and clear examples with exact solutions inevitability of black holes in GR has been strictly proved. For real stars with the account of interactions the collapse with formation of black hole has appeared also inevitable at star's mass of 3-4 solar mass. These elementary facts of long time established science I remind you only for spoken about the same things. Otherwise, I feel, you now will obscure all, having as starting point some discrepancies of definitions and on it will make a fuss and hang to me noodles on ears.

Sagredo. To make a fuss and noodles on ears are classical attributes erroneous, but temporarily won paradigms - they avoid exact definitions and a boring logic and quickly pass to puzzled phantasmagorias exciting imagination. But, I will not be off your guard by unnecessary specifications. I only will make last necessary specification and then we will take occasion by the forelock - you will prove to yourselves that in GR black holes are forbidden and the collapse leads only to formation of frozars - normal stars, but with a gravitationally frozen internal structure. You not only will come to this conclusion, but also will be surprised, how someone cannot understand this simple and absolutely obvious fact.

Salviati. Here also has tritely gone - are you sense that your roof somewhere brings. I have already mentally prepared a grave for these yours frozars and have thought up what there will be written: «Here is grounded frozar from a core of quasar which has died, before been born at all». About forelock you have already told, I can wait, while they is cast away also hoofs. Then already, I hope, we will sing also a farewell hymn. So, here I still will suffer last your specification, but no more.

Sagredo. That on carelessness you prepare for frozars, is required for your favorite black holes though by idea they are an ideal grave for everything. By the way, black holes would be inevitable, if the Newtonian physics could holds in strong gravitational fields too. But in strong fields it is fair only GR and, as below it will be convinced, this theory leads only to frozars. So I will define my last specification.

Salviati. Only do not pull - remember, time for you already finished and my patience too.

Sagredo. In science success is a result of patience of thought as something was say still Newton. Breathe deeper. When we will reach to frozars and you will understand how they are formed, I am confident; you will feel such sense of relief that your patience will be satisfied.

Salviati. A little more and I will suffer not on shortage of air, but I will burst from anger.

Sagredo. Impatience at the criticism is symptoms of mistake. By the way, it is exact enough diagnosis, so concentrate and calm down. So, we specify our points of view.

\section{General relativity really leads only to frozars? It is interesting!}

Sagredo. You agree that in the black hole theory collapse into the gravitational radius is not observable for external observers, and lies, as written in textbooks, behind the temporary infinity of world time, but before that occurs it is simple and even ordinary. A dust shell is very successful example since here all is precisely known. At falling of thin dust shell in own gravitational field, at closely approaching the gravitational radius, proper time and the falling process of the shell are slowed down with respect to world time, so that for external observers the dust shell becomes forever frozen over the gravitational radius. In empty space inside this shell the test particle's proper time also become frozen forever in terms of world time and the test particles will be frozen at the those places where they have appeared before freezing. 
Agree with this specification?

Salviati. Yes, in terms of world time the first stage of collapse is described right, though in proper time the picture looks absolutely different. I asked you to consider a dust ball too. There depict your last, more likely, extreme specification also and then we will look, where and on what banana peel you have slipped.

Sagredo. Yes, of course, now I pass to a dust ball too. When the ball is contracted because of own gravitation and a particle freely falls in in a direction to ball's centre, as surface approaches the gravitational radius, delay of proper times in terms of world time reaches such high degree that the surface quickly freezes over the gravitational radius. According to exact solutions of the Einstein equations of GR [Tolman (1934, 1939), Oppenheymer and Snyder (1939)], the proper time delay in internal layers is stronger, than on surface and it is the strongest at the centre, thus the centre and other layers freezes before surface.

Salviati. I did not read anywhere that during collapse in terms of world time the centre freezes before surface. It is exact? You checked up under known solutions? Always it was intuitively seems to me that at collapse the interior levels fast fall to the centre!

Sagredo. Yes, certainly checked up and it is surprisingly. Though in those known solutions this fact contained initially, anybody did not pay attention. All these are consequences of GR when we model the process by the equations of GR and their solutions and epoch by epoch in world time trace positions of layers of the dust ball, starting at time when gravitation was not so strong. We mark positions of layers at each moment of world time (epoch) and indications of standard clocks on them, the intervals of proper time showing the increasing dilation and freezing about certain moments of proper time in process of ball's contraction. This boring picture of freezing of proper times of layers of the ball in world time is a strict consequence of GR and it takes place in the black hole theory too at the first stage of collapse when ball's surface on proper time is still outside the gravitational radius. Yes?

Salviati. Yes, this picture really very boring, unsuccessful and distorting the dynamics of process an image of the first stage of stellar collapse. But it only a prelude to a true collapse which becomes dynamical and interesting as soon as you will pass to more successful coordinates revealing a true picture of contraction in proper time, especially, at the second stage of collapse where they are already irreplaceable.

Sagredo. So, you agree, that for the first stage of collapse while all is described correctly?

Salviati. While, of course, yes. But you still in any way do not concern and avoid the second stage of collapse and those moments of proper time when the dust shell or surface of the dust ball crosses their gravitational radii and all fails to the centre.

Sagredo. Thus, we have come to the full harmonization of descriptions in two treatments of the first stage of collapse, i.e. until when surface supposedly passes the gravitational radius. I congratulate you, now you in one step to be a follower of the theory of frozars.

Salviati. To tell the truth, I have not noticed it and in any way I do not feel that my beliefs in something have changed. When you will be in one step to be a camel, I too will warn you precisely also and you will say that it do not feel!

Sagredo. Turning to a camel is your imagination, while even strictly following to GR I must to prove that I am not a camel, is a reality. But when the reality is unhappy, it doesn't give way to despair, to remain as optimist and to make efforts for improving it and then the reality will change for the better.

Salviati. Really? And you can set an example to such transformation of the reality by efforts 
of your thought only? Now it is clear, where we will be ended by yours frozars!

Sagredo. And at least on your example. I have told, even now you think that you are a follower of the conventionally accepted black hole theory and, consequently, with quiet conscience feel comfortably in the scientific community where only this concept is identified with a serious science, consider yourself have a right to use all blessings of civilization in the status of deserved and, consequently, the dear and known scientist, recognized as that by other dear scientists. And now, believe to me, when we will leave, all it will not be for you - you will be convinced that from GR follow not black holes, but frozars and, consequently, at once become in opposition to all scientific community. Then you cannot participate in that international conference where you are invited to report about applications of the concept which you will not consider any more correct, you will not publish and teach lectures on black holes and still it is not known, how all it will effect on your career and welfare. All these are examples of the transformation your reality. How it is pleasant to you?

Salviati. It is unpleasant not that you predict for me, but how you are self-confident, thinking that you can manipulate my visions which have not changed in any way during our conversation and, I think, it does not threaten for me in the foreseeable future. So, already you begin to invent phantasmagorias. But, it is time to me; I said you that your time has expired and mine especially. Good-bye. You frozars will manage and without me.

Sagredo. It is time to me too, so, good-bye. As I have almost reached my aim since you already appreciated that the theory of frozars corresponds GR.

Salviati. Really? What you say? When I appreciated that? I have not understood what this theory is and what a fruit is frozar. So, do not go too far and do not attribute me that you so would like to hear.

Sagredo. I have already got definition of this theory and you were convinced that it is very simple. The description of the first stage of stellar collapse in frameworks of GR in terms of world time is the theory of frozars. A star at certain finite world time moment with gravitationally frozen layers is frozar. At any world time moment corresponding proper time moments on star's surface and internal layers appear almost frozen at some finite values different for different layers. Thus, the centre freezes before surface at a little smaller value of proper time. That is all what I wanted to explain!

Salviati. Well, if you simply give a new name for a picture of the first stage of collapse, which is known and quite obvious for a long time, you can simply say that, instead to raising it to a rank of a new theory. But I have not understood where you have put the second stage when star's surface crosses the gravitational radius in proper time and falls to the centre - the description of this second stage is essence and main achievement of the black hole theory.

Sagredo. Here the essence of the theory of frozars, why the new name is entered, is that here it is firstly proved that in GR this second stage of stellar collapse is absent and collapse is entirely reduced only to its first stage. A thin dust shell and a dust ball, surface of which is very close to their gravitational radius, at any moment of world time are in the frozar state with a frozen internal structure and further no anything happens with them. That moment of proper time when the second stage of collapse should begin apparently never comes, since proper time on the surface is gravitationally frozen with respect to world time, representing the regularly lasting evolution time of the Universe as whole. The moments of proper time are strongly restricted by the world time moments.

Salviati. Thus, you declare that the former theory of black holes should be divided on two parts - one part describing the first stage of collapse to be named as the theory of frozars and 
to consider as a unique realistic application of GR, and the second part describing the second stage of collapse to be throw out as a mathematical abstraction. Yes?

Sagredo. Quite right. You at last have started to think clearly and soberly.

Salviati. While I only formulate your statements and I do not agree with the statement about absence of the second stage. So, what stops collapse - simply the freezing in terms of world time? It was well known earlier.

Sagredo. Yes. The star is embedded into the real Universe where average cosmological time is going practically uniformly and on the star, according to GR, there is a proper time dilation with respect to this cosmological time due to strengthening of gravitation at contraction. As a result, collapse stops naturally due to the gravitational freezing of all processes on surface and internal region, with respect to the regularly proceeding cosmological time. So those black holes which were predicted by the Newtonian theory, in frameworks of GR can be formed never. According to GR in the real world at any world time moment at least the surface of any star coexists simultaneously with other objects. At any moment of this real history of the Universe, or at given epoch, a dust ball, surface of which has been frozen outside the gravitational radius, is frozar with a frozen structure in entire volume.

Salviati. I then specify - that you say, is external manifestation of collapse in world time and this picture is limited because a world time does not cover all worldline of falling particles since this system of coordinates is not full. In fact nothing stops a further collapse in terms of proper time for falling observers on surface! This time for them does not freeze! If from their point of view the fact that the temporary evolution of other world is simply strongly fastened does not means that they will not continue falling to star's centre, so the second stage of collapse is inevitable. I said that all of you will reduce to overestimate of a role of some special choice of coordinates and will assert that only it is true and that therefore there is no leaving under horizon.

Sagredo. Do not hurry up to do hasty conclusions.

\section{General relativity leads only to frozars. It is really obvious!}

Sagredo. To clear yours mind of last doubt, sorry, extreme doubt, let us look at collapse also from the point of view of observers falling on star's surface. You have told that from their point of view all local processes proceed normally, but only events in all other world begin to occur in much fastened rate. Thus, you and your observer, as a rational people, not inclined to overstate your role in destiny of other world, well understand that in all huge Universe's the average rate of cosmological world time cannot be fastened more and more in process of falling of some layer of some star. Therefore you will interpret that as a gravitational delay of local proper times of falling observers with respect to regularly proceeding world time. Yes?

Salviati. Yes, it is right. The relative delay obviously and an enough competent falling observer, most likely, will interpret it as the delay of its proper time with respect to the cosmological time. Well, and what from this? Here we say not about interpretation, but that the course of its proper time proceeds independently as quickly evolves other world. So, I am right also and all of your proof is reduced to a private opinion of someone.

Sagredo. Here you have not paid attention to the main fact which I have emphasized earlier. World time and proper time on star's surface are strongly related with each other. In GR both times mark the same events along the same world line of each particle. To each instant of one of them there corresponds a certain instant of another - there an event is the same. And so, then that moment of proper time on surface, when your observer easily could cross 
gravitational radius in former theory, really will come only after the infinitely far future of the real world. This mathematical expression in translation into the physical language means that in the real world this moment will not come never for the falling observers too. They will be forced to wait, while the Universe will really live this infinite world time, which by definition will not come to the end at a given time. You understand it? So, are you agreeing that in GR both kinds of times are strongly related with each other?

Salviati. I understand it and I know that two times are related when describe the same events. But you are right that surface of real collapsing objects yet have not reached a gravitational radius. They are named as collapsing objects since their horizon was not formed yet or, even more carefully, astrophysical black holes. It is well known fact.

Sagredo. We have agreed that we will follow to standard GR. So, then tell directly - at real collapsing objects horizon not only does not formed yet, but it will not be formed never since you cannot indicate a concrete world time moment of its formation. At any moment of the world time, marking epochs of the real world, a surface of your object will be outside of its gravitational radius, the internal structure will be frozen and exactly the same as it was directly before last photons escaped its surface. Here such object, which you at last have started to admit, is frozar. It is really well known and most of cautious astrophysicists are adhering such point of view. But at discovering of each such real object hotheads confirm to all world and approve that the next black hole is discovered. There, showing the Newtonian picture of collapse, they declare it as a strict consequence of GR.

Salviati. So, apparently, your position is completely clear to me. The theory of frozars, as that part of the black hole theory which is restricted by the first stage of collapse, describes the astrophysical black holes at which a horizon does not formed yet and which earlier have been named as collapsing objects, and you shortly name them as frozars. And what, these banalities you cannot tell me right at the beginning? I could be agreeing with it.

Sagredo. So, you recognize that those thousands of already discovered compact objects, which posed as black holes, in fact, directly saying, are not the such objects and they are the collapsing objects or frozars with normal, but frozen on different distances from their centre particles and, mainly, that there is no horizon and singularity at the centre

Salviati. Well, in general yes.

Sagredo. And also recognize that they never will turn to black holes?

Salviati. In world time - yes, will not turn.

Sagredo. And in what time turn - in proper time?

Salviati. Under the black hole theory - in proper time. But, as you have told, in GR these moments of proper time in our world will not come never because of the real freezing of proper times in each compact object. If so, yes, never will turn to black holes.

Sagredo. In some popular textbooks it has been wrote that freezing of star's surface is an optical effect caused by slowering of light velocity near the star. You understand that it is a mistake? Are you agree, that freezing with respect to world time is absolute and all observers with any coordinate can compare these two times expressed in their coordinates and can check that star's surface is frozen with respect to the cosmological world time? The same indicate the coordinate clocks on surface too, going synchronously with clocks of distant observers.

Salviati. Yes, certainly. It is definitely not an optical effect and by using the coordinate clocks going synchronously with clocks of distant observers, it is really possible to define 
simultaneity of events on surface with distant events. I, apparently, start to understand importance of definition of the simultaneity of events on surface and on removal. It turns out that as soon as we recognize this physical simultaneity of events, a world line of surface in any moment of cosmological time cannot cross the gravitational radius. Then all particles in the star remain ordinary and simply frozen at different places and by the star really occur nothing? Then really there is no place for the second stage of collapse ..., there will not be horizon, no singularity? It is wonderful! While it is little shock for me... I should conceive that...

Sagredo. You only do not worry and if still there are any doubts or arguments, spread them for not to decide and not to come back again to with what have begun.

Salviati. How I cannot worry? I shake by this! Such simple mistake is really admitted? At the basis of such grandiose paradigm, as black holes? No, I cannot believe that you are right... Mainly, it cannot be! So, we will return and give once again easier and, mainly, strictly prove, why in GR the second stage of collapse is absent?

Sagredo. I will make it with pleasure. Definitively to be convinced of freezing of a dust ball with transformation into frozar and in absence of the second stage of collapse, you can take exact or numerical solutions of the equations of GR and look after how change in world time the perimeter of surface and internal layers. Then clearly will see that before the surface will closely approaches the gravitational radius, a proper time rate at the centre practically stops and there all freezes, then this freezing quickly reaches up to surface. After that world lines of particles of the dust ball forever remain parallel to each other on that distance from the centre on which they were as freezing was started. Are you imagining it?

Salviati. Yes, I am imagining. They will be really parallel and how it is misfortune! I always imagined that star's interior quickly fails to the centre and that we do not receive the information about it only. Really, we can model all that by the equations of GR in terms of world time, considering sets of simultaneous events on removal, on surface and in the ball! So, if all it gives only the transition into a frozar state with parallel world lines of particles in entire volume and no anything else occur, I am understand. Simply improbably! Interestingly, what will change if to include pressure and temperature?

Sagredo. Contraction of a dust ball finishes with the transition into the frozar state and no more anything. If in more realistic models to include other interactions of particles, the contraction will lead to growing of pressure and temperature and all depends on the equation of matter, relating density, pressure and temperature. Then the transition into the frozar phase may not take place - the star can overheat and stops, starts to pulsate or can expand up to explosion at the final stage. Such realistic process of modeling on the basis of GR also is the theory of frozars. Now you can tell to yourselves, notice, not to me, are you accept the theory of frozars?

Salviati. Unfortunately, while it seems yes. I say while since I still should reconsider all that.

Sagredo. It is glad for you that could overcome yourself and meanwhile though shallow this bitter pill. I wish pleasant digestion and the further sobering up.

Salviati. Though that we will pull, when answers only two - you are right or not. So, let us suppose that after I will think overall and read the literature, I will agree with this new treatment. I repeat, while only we suppose that. Then what from this follows? What changes in GR, astrophysics and what from the former black hole theory remains? You can shortly give answers to these questions? 
Sagredo. Certainly, you should think over all again and I am not put pressure. You specialize on it, have devoted a part of your life and it will be not simple to you to see, how some of your works on your eyes will devalue, if do not turn to garbage. But, the science moves ahead by the trial and error, so not too worry and simply move on new and more right way. I after all developed also threw out many versions, while some years I am convinced of correctness only this theory which initially was not pleasant to me too.

Salviati. Do not advance events; I am not so confident yet. Pass to consequences and then I will look. A correct theory always leads to unexpected both cardinal improvements and new predictions, so to these signs it will be already visible, will your theory pull for this role or not. So, what we receive, if we reject black holes and accept your frozars?

Sagredo. Remember as you say about greatest crisis hidden under horizon? So, according to the theory of frozars, a horizon and singularity do not exist and there no a crisis, even hidden. In GR a point source do not exist at all - in general these no objects of size less than their gravitational radius. In astrophysics it solves a problem of collapse both compact stars and galaxy nuclei, including quasars. One of dissimilarities of frozar from black hole - under certain conditions, i.e. at fast growth of temperature and pressure, contraction can turn to expansion up to explosion at a final stage with releasing much more matter and energy, than it was known till now. Thus, for frozars the collapse, generally, is reversible.

Salviati. Really? How large should be mass of collapsing object when temperature at its centre can grow so that it may leads to expansion with explosion at the final stage?

Sagredo. There it is required to study already not dust ball, but more realistic models. In astrophysics explosions of different scale have been observed, energy source of which are while is unknown and now the theory of frozars allows modeling such processes, including the reversing of collapse, or anticollapse, as probable sources of such most powerful ejections of radiation and matter. Such objects which at collapse had not time to run into the frozar state and have started to be refrozen, in the given approach it is proposed to name as glowsars, from words glow star.

Salviati. Really, many problems become simpler! If GR further admits recollapse, then unlimited prospects will open - now it is difficult to say how large these prospects. Thus, if a real collapsing star does not freezes, it turns to a glowsar state, as you say, with some activity? But, how it is proved?

Sagredo. In the idealization of the dust matter, the formation of frozar is a strong consequence of GR. But what can pass into the glowsar state depends on interactions, state equations and stellar parameters. GR only admits the formation of glowsars, so it is meaningful to study their properties and manifestations while assuming that in some cases they can be realized and be observed.

Salviati. Well, at least, the observation of these glowsars is not as hopeless as frozars, so it inspires some optimism. Are there other interesting consequences of the new theory?

Sagredo. Not less important that the new theory of relativistic compact objects solves the problems with infinitely high energy fluctuations in particle physics - there should be quanta with the Planck energy - frozons, and particles with higher energy, according to GR, do not exist. Gravitational redshift of frequencies near a source of such quanta with the same size puts a top limit to their frequencies - particles with higher energy cannot be emitted by any source. Quantum gravitation, which does not been constructed till now, as a result, can be easily constructed due to the sufficiently simplification of the theory at such restriction. These are rather cardinal improvements which do unnecessary many of the hypotheses which have 
been put forward in particle physics during last decades.

\section{Epilogue}

Salviati. If it so it is very tempting. We do not discuss anything about them.

Sagredo. We will discuss at another time. Have not forgotten, it is time to you on conference on black holes.

Salviati. Yes, really, there I will present a large report and then I will receive a big grant.

Sagredo. In the report you will tell something about frozars too?

Salviati. You in the mind! I cannot be at war with entire world! But here when that your author will be supported by scientists leading in this area, I will surely support him too.

Sagredo. Yes, of course, and thanks on those. I have very tired you, are you want for a discharge one eastern fable.

Salviati. Fine, tell please!

Sagredo. An uncle of Hodja Naseriddin in a far city has inherited him big money, but he did not have money to such long trip and taking all that. He has come to neighboring rich man and has asked him about loan in ten gold moneys for trip to reach and come into the inheritance in ten thousand gold moneys. That has told that he will support, but under one condition: "You there go, receive your ten thousand gold moneys and then, after returning, I will surely lend you ten gold moneys".

Salviati. Yes, the east is specifically and at ancient epochs there was another culture!

Sagredo. Scientific community's culture, as you show, now like that...

Salviati. M-yes. Give me please an article about frozars. I will read in details and send it to ten known experts who know me and at conference I will explain it him. This will be my ten gold moneys. O key?

Sagredo. O key. Now you use by own head and on its direct mission! 